\title{
SUSCEPTIBILITY OF S.AUREUS TO METHYLENE BLUE HAEMATOPORPHYRIN, PHTALOCYANINES PHOTODYNAMIC EFFECTS
}

\author{
Tzvetelina Gueorgieva1, Slavcho Dimitrov1, Violeta Dogandhiyska1, Vasil \\ Kalchinov1, Marieta Belcheva1, Vanya Mantareva2, Ivan Angelov2, Veselin \\ Kussovski3 \\ 1)Department of Conservative and Pediatric Dentistry, \\ Faculty of Dental Medicinep Medical University - Sofia, Bulgaria \\ 2)Institute of Organic Chemistry, \\ 3)Institute of Microbiology, Bulgarian Academi of Sciences - Sofia, Bulgaria
}

\section{SUMMARY}

Introduction: Many of oral diseases are caused by microorganisms. The emergence of antibioticresistant strains, such as methicillin-resistant Staphylococcus aureus and vancomycin-resistant Enterococcus faecalis, stimulated a search for alternative treatments. Such an alternative method is photo-activated disinfection. The aim of this study is to compare the antimicrobial properties of four new photosensitizers-methylene blue, haematoporphyrin and new $\mathrm{Ga}, \mathrm{Zn}$ - phtalocyanines after the laser radiation $(630 \mathrm{~nm})$ against Staphylococcus aureus.

Materials and methods: For the study gram-positive Methicillin-resistant Staphylococcus aureus 1337 from collection of the Institute of Microbyology, Bulgarian Academy of Science is used. For PAD is used source of light which activates photosensitizer trough emission of visible light at a specific wavelength. Suspensions of Staphylococcus aureus was irradiated with diode laser $(630 \mathrm{~nm})$ in the presence of methylene blue, haematoporphyrin and new $\mathrm{Ga}, \mathrm{Zn}$ - phtalocyanines. There were two control groups: samples that were only treated with dye and samples that were exposed neither to the laser light nor to dye.

Results: Reduction in the viability of Staphylococcus aureus was achieved with some of the photosensitizers and light associations.

Conclusions: Photoactivated disinfection appears as an effective method of Staphylococcus aureus inactivation.

Key words: photodynamic therapy, photo-activated disinfection, microbial resistance, photosensitizer, antimicrobial action .

\section{INTRODUCTION}

Many of oral diseases are caused by microorganisms. Traditionally impact on aerobic and anaerobic oral cavity microorganisms is being performed through mechanical cleaning and chemical treatment by agents with various antimicrobial activities. In order to affect the pathogens in radical-canal system sodium hypochlorite, chlorhexidine gluconate, hydrogen peroxide, $15-17 \%$ EDTA, 3-10\% citric acid, calcium hydroxide, metronidazole, are being applied, as well as combinations of these $/ 11 /$. In recent years, the emergence of antibioticresistant strains, such as methicillinresistant Staphylococcus aureus and vancomycin-resistant Enterococcus faecalis, stimulated a search for alternative treatments. Such an alternative method is photodynamic therapy - PDT. /8, 14/. Photodynamic therapy (PDT) is basically applied in medicine for treatment of cancer $/ 1 /$. PDT involves three components: light, a photosensitizer, and oxygen. A photosensitizer or its metabolic precursor is administered to the patient. Upon irradiation with light of a specific wavelength, the photosensitizer undergoes a transition from a low-energy ground state to an excited singlet state. Subsequently, the photosensitizer may decay back to its ground state, with emission of fluorescence, or may undergo a transition to a higher-energy triplet state. /7, 9 /. The triplet state can react with endogenous oxygen to produce singlet oxygen and other radical species, causing a rapid and selective destruction of the target tissue. There are two mechanisms by which the triplet-state photosensitizer can react with biomolecules. Type I involves electron/ hydrogen transfer directly from the photosensitizer, producing ions, or electron/hydrogen removal from a substrate molecule to form free radicals. $/ 1,7,9,14 /$. These radicals react rapidly with oxygen, resulting in the production of highly reactive oxygen species (superoxide, hydroxyl radicals, hydrogen peroxide). Type II reactions produce the electronically excited and highly reactive state of oxygen known as singlet oxygen. /1, 5, 7, 9, 14/.

In PDT, it is difficult to distinguish between the two reaction mechanisms. PDT requires a source of light that activates the photosensitizer by exposure to low-power visible light at a specific wavelength. /1, 5, 7, 9, 14/. Most photosensitizers are activated by red light between 630 and 
$700 \mathrm{~nm}$, corresponding to a light penetration depth from 0.5 $\mathrm{cm}$ (at $630 \mathrm{~nm}$ ) to $1.5 \mathrm{~cm}$ (at $700 \mathrm{~nm}$ ). /6/. At present, diode laser systems that are easy to handle, portable, and cost-effective are used predominantly.

The principle of photodynamic therapy lies in the heart of photo-activated disinfection - PAD. Photosensitiser molecules attach to the membrane of the bacteria . Irradiation with light at a specific wavelength matched to the peak absorption of the photosensitiser leads to the production of singlet oxygen, which causes the bacterial cell wall to rupture killing the bacteria. /7, 13, 14/. Microorganisms that are killed by PAD include bacteria, fungi, viruses, and protozoa. The development of resistance to PAD appears to be unlikely, since, in microbial cells, singlet oxygen and free radicals interact with several cell structures and different metabolic pathways. /3, 7/.

Many natural and synthetic photoactive compounds have photosensitizing potential. Photosensitizer should be non-toxic, and should display local toxicity only after activation by illumination. The majority of the sensitizers used clinically belong to dyes. Initially antimicrobials dyes included tricyclic dyes such as acridine orange, methylene blue, fluorescein, Rose Bengal /4/. An inherent problem in using tricyclic dyes is that they might be toxic or may act as mutagens. Our project is directed to the development of new biomaterial for PAD (the so called photosensitizer), which is based on the pairing between a photoactive tetracyclic photosensitizer, e.g. phthalocyanine with the second photosensitizer as fullerene incorporated in liposome vehicles. The new biomolecule conjugates are expected to be selective towards bacterial cells, i.e. increasing microbial phototoxicity.

The aim of this study is to compare the antimicrobial properties of four photosensitizers - methylene blue, haematoporphyrin and new $\mathrm{Ga}, \mathrm{Zn}$ - phtalocyanines after the laser radiation $(630 \mathrm{~nm})$ against Staphylococcus aureus. Staphylococcus aureus is etiological factor of periodontitis, refractory endodontic lesions and takes part in infectious endocarditis after bacteraemia, caused by dental manipulations $/ 2,10,12 /$.

\section{MATERIALS AND METHODS}

For the study gram-positive Methicillin-resistant Staphylococcus aureus 1337 from collection of the Institute of Microbyology, Bulgarian Academy of Science is used . For PAD is used source of light which activates photosensitizer trough emission of visible light at a specific wavelength. Suspensions of Staphylococcus aureus was irradiated with diode laser $(630 \mathrm{~nm})$ in the presence of methylene blue, haematoporphyrin and new Ga, Znphtalocyanines. There were two control groups: samples that were only treated with dye and samples that were exposed neither to the laser light nor to dye. From each sample, serial tenfold dilutions were prepared and aliquots of $0.1 \mathrm{ml}$ of each dilution were plated in duplicate on Muller Hunton agar. After incubation at $37^{\circ} \mathrm{C}$ for $24 \mathrm{~h}$, the number of colony-forming units $(\mathrm{CFU} / \mathrm{ml})$ was obtained.

\section{RESULTS}

Reduction in the viability of Staphylococcus aureus was achieved with some of the photosensitizers and light associations. In order to determine the optimal concentration of photosensitizer required to effectively kill the oral pathogen Staphylococcus aureus, 5 mikrom of each photosensitizer and times of irradiation were tested. For S. aureus the irradiation with diode laser in presence of Gaphtalocyanines for 12 and 20 minutes lead to - 0,1 CFU/ml viable MRSA; for 5 minutes - $10 \mathrm{i} \mathrm{CFU} / \mathrm{ml}$ viable MRSA. In presence of $\mathrm{Zn}$-phtalocyanines - for 5 minutes there were - 10i CFU/ml viable MRSA; for 12 and $20 \mathrm{~min}$ respectively $10 \mathrm{I}$ and $1 \mathrm{CFU} / \mathrm{ml}$ viable MRSA. The use of Methylene blue for 12 and 20 minutes gave 10i CFU/ml viable MRSA; for 5 minutes there was poor reduction of S. aureus - $105 \mathrm{CFU} /$ $\mathrm{ml}$ viable MRSA. The irradiation in presence of Porphyrin leads to - $107 \mathrm{CFU} / \mathrm{ml}$ viable MRSA.

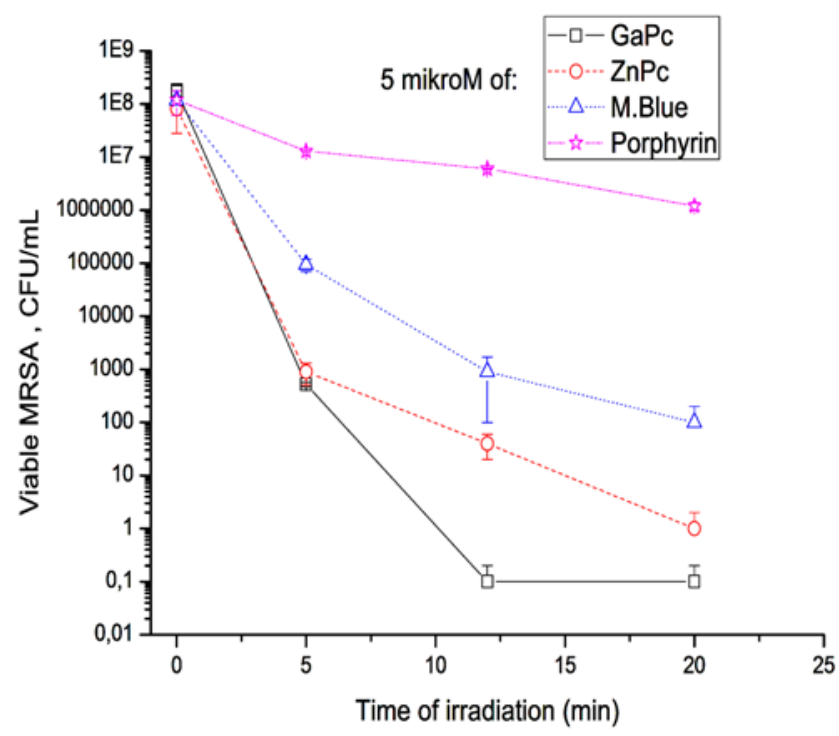

Photodynamic effect of GaPc, ZnPC, MB and Porrphyrin on MRSA depending on time of irradiation

From obtained results we can conclude that the irradiation of Staphylococcus aureus with diode laser in presence of Ga-phtalocyanines more then 12 minutes and in presence of Zn-phtalocyanines for $20 \mathrm{~min}$. is sufficient to kill the bacteria and lead to complete yeast destruction .In presence of $\mathrm{Zn}$-phtalocyanines and of Methylene blue for 12 and 20 minutes, there were a great reduction of S. aureus. The use of Methylene blue for 5 minutes and of Porphyrin for 5, 12 and 20 minutes weren't very effective to destroy the microorganism. 


\title{
CONCLUSIONS
}

Photoactivated disinfection appears as an effective method of Staphylococcus aureus inactivation. The application of new phtalocyanines leads to complete yeast destruction. The proper combination of drug-light doses can lead to a full photodynamic response without bacteria survival. The proposed method could become a promising alternative treatment of Staphylococcus aureus infections in dental medicine.

Acknowledgements: This work is supported from the Grants DO-02-177/Dec. 2008 of the National Science Fund, Sofia, Bulgaria

\section{REFERENCES:}

1. Dougherty Thomas J., Charles J. Gomer, Barbara W. Henderson, Giulio Jori, David Kessel, Mladen Korbelik, Johan Moan, Qian Peng. Photodynamic Therapy. Journal of the National Cancer Institute. 1998; 90:889-905

2. Fernбndez Guerrero ML, Gonzбlez Lypez JJ, Goyenechea A, Fraile J, de Gyrgolas M ; Endocarditis caused by Staphylococcus aureus: A reappraisal of the epidemiologic, clinical, and pathologic manifestations with analysis of factors determining outcome; Medicine (Baltimore).; 88: 1-22 ; 2009

3. George Saji and Anil Kishen . Influence of Photosensitizer Solvent on the Mechanisms of Photoactivated Killing of Enterococcus faecalis. Photochemistry and Photobiology. 2008; 84: 734-740

4. Gil-Tomas J, Tubby S, Parkin PI,
Narband N, Dekker L, Nair PS, Wilson M, Street C. J Mat Chem 17: 3739 ; 2007

5. Hsi R. Alex, David I. Rosenthal and Eli Glatstein. Photodynamic Therapy in the Treatment of Cancer. Current State of the Art. Drugs 1999;57: 725-734

6. Komerik N, MacRobert AJ. Photodynamic therapy as an alternative antimicrobial modality for oral infections. J Environ Pathol Toxicol Oncol. 2006;25:487-504

7. Konopka K. and T. Goslinski. Photodynamic Therapy in Dentistry. J Dent Res . 2007;86:694-707

8. Korbelik Mladen, PhD. PDTAssociated Host Response and its Role in the Therapy Outcome. Lasers in Surgery and Medicine 38:500-508; 2006

9. Macdonald Ian J. and Thomas J. Dougherty. Basic principles of photody- namic therapy. J. Porphyrins Phthalocyanines 2001; 5: 105-129

10. Persson GR, Imfeld T; Periodontitis and cardiovascular disease; Ther Umsch.; $65: 121-6 ; 2008$

11 Radeva E., B. Indzhov; Microbiological characteristic of endodontium. Agents used for root canal microflora treatment. Stomatology, 81: 21-26; 1999

12. Reader CM, Boniface M, BujandaWagner S; Refractory endodontic lesion associated with Staphylococci aureus ; J Endod.; 20:607-609; 1994

13. Wainwright Mark. Photodynamic antimicrobial chemotherapy (PACT). Journal of Antimicrobial Chemotherapy. 1998; 42: 13-28

14. Walsh LJ. The current status of laser applications in dentistry. Australian Dental Journal 2003; 48:146-155

\author{
Address for correspondence: \\ Dr. Tzvetelina Gueorgieva \\ Department of Operative Dentistry and Endodontology \\ Faculty of Dental Medicine - Sofia \\ 1, Sv. Georgi Sofiiski bul., 1000 Sofia, Bulgaria \\ e-mail: cvettyg@gmail.com
}

